\title{
Delaying Seed Germination and Improving Seedling Fixation: Lessons Learned During Science and Payload Verification Tests for Advanced Plant EXperiments (APEX) 02-1 in Space
}

\author{
Jin Nakashima ${ }^{1}$, J. Alan Sparks ${ }^{1}$, John A. Carver, Jr. ${ }^{2}$, Shawn D. Stephens ${ }^{3}$, Taegun Kwon ${ }^{1}$, and Elison B.
} Blancaflor $^{1}$

${ }^{1}$ Plant Biology Division, The Samuel Roberts Noble Foundation, Ardmore, OK $73401 ;{ }^{2}$ Kennedy Space Center, Test and Operations Support Contract, Kennedy Space Center, FL 32899; ${ }^{3}$ Kennedy Space Center, Engineering Services Contract, Kennedy Space Center, FL 32899

\begin{abstract}
Here we report on the science verification test (SVT) and the payload verification test (PVT) that we conducted in preparation for experiments evaluating the impact of microgravity on Arabidopsis thaliana root development and cellular structure. Hardware used for these experiments was the Advanced Biological Research System (ABRS) and Kennedy Space Center (KSC) fixation tubes (KFTs). A simple procedure to delay seed germination prior to installation on ABRS involved the construction of a metal box with a single far-red (FR) lightemitting diode (LED). The exposure of Petri dishes containing seeds (ecotype Columbia) to FR
\end{abstract}

Key words: Advanced Biological Research System (ABRS); Science Verification Test (SVT); Payload Verification Test (PVT); KSC Fixation Tubes (KFTs); Arabidopsis thaliana; Far-red Light; Microscopy; Germination

Correspondence to: Elison B. Blancaflor The Samuel Roberts Noble Foundation Plant Biology Division 2510 Sam Noble Parkway Ardmore, OK 73401 Telephone: 580-224-6687 E-mail: eblancaflor@noble.org light immediately after planting and maintaining Petri dishes in the dark prevented seed germination until exposure to white light on ABRS. Additional tests revealed that germination can be delayed for up to 10 weeks with FR light treatment. Seedlings fixed in KFTs preloaded with glutaraldehyde for subsequent microscopy studies were not adequately preserved. We suspected that poor fixation was due to the extended contact of glutaraldehyde with oxygen while stored on KFTs, which likely contributed to fixative oxidation. During PVT, minor modifications to address fixation problems encountered during SVT included storing KFTs with glutaraldehyde at $4^{\circ} \mathrm{C}$ in the dark, increasing glutaraldehyde concentration from $3 \%$ to $5 \%$, and bubbling nitrogen $\left(\mathrm{N}_{2}\right)$ gas over the glutaraldehyde solution prior to loading the KFTs. These changes led to improvements in the quality of microscopic images. Lessons learned from SVT and PVT allowed us to optimize some of the preflight protocols needed to successfully implement Advanced Plant EXperiments (APEX) in space.

\section{INTRODUCTION}

For many years, the National Aeronautics and Space Administration (NASA) and other international space agencies have had an emphasis on research to understand the basic mechanisms by which plants develop in the microgravity 


\section{Lessons Learned from APEX 02-1 SVT and PVT}

environment of low Earth orbit. The expectation is that knowledge gained from this type of fundamental research will help guide the design and implementation of future advanced life support systems for long-term exploration of space beyond low Earth orbit (Ruyters and Braun, 2014). The resources and planning that go into a plant space biology experiment can be a daunting task and, given the scarcity of spaceflight opportunities, months and sometimes even years of preparation are needed to ensure success. The SVT and PVT are essential components of NASA-sponsored spaceflight research. During SVT and PVT, investigators are given the opportunity to conduct high fidelity testing to determine how their chosen model organism performs on approved spaceflight hardware, and to critically evaluate experimental procedures that will be used for preflight and post-flight operations. Results from SVT and PVT then form the basis for determining whether experiments are ready for spaceflight, as judged by predefined success criteria. SVT and PVT outcomes can also be used by investigator teams in consultation with NASA support personnel to implement minor adjustments in experimental protocols prior to the actual spaceflight.

Despite valuable insights gained from SVT and PVT, some results from these tests are usually known only by the investigator team and NASA personnel assigned to assist in the implementation of the spaceflight experiments. This is unfortunate because lessons learned during SVT and PVT can provide a useful resource to other researchers who are at an early stage of preparation for spaceflight or guide those who are considering spaceflight opportunities to address their biological process of interest. We recently completed the SVT and PVT for our second plant space biology experiment originally designated as APEX 02-1 in space. The goal of this space biology project was to better understand plant development in microgravity, focusing on the interplay between the cytoskeleton and cell wall architecture in the regulation of root growth orientation using Arabidopsis thaliana as a model plant.

APEX 02-1 built on our first spaceflight studies that used Biological Research in Canisters (BRIC-16) hardware on the STS-131 mission (Nakashima et al., 2014). For APEX 02-1, we planned to use the ABRS housed on the
International Space Station (ISS) (Levine et al., 2009) to test the hypothesis that microgravityinduced transcriptional reprogramming of cellwall-related genes defines root skewing behavior previously observed in microgravity (Millar et al., 2011; Paul et al., 2012; Nakashima et al., 2014). In this paper, we report on certain aspects of APEX 02-1 SVT and PVT, focusing primarily on methods to delay arabidopsis seed germination prior to installation on ABRS and microscopic analyses of seedlings grown on ABRS and fixed in KSC KFTs (Paul et al., 2005). We expect that some of the issues documented in this paper would benefit other plant space biology researchers who plan to use similar spaceflight hardware and procedures for their experiments.

\section{MATERIALS AND METHODS}

\section{Arabidopsis Seed Processing, Planting, and Far-Red Light Treatment}

The SVT was performed from October through November 2012, and the PVT from February through March 2013 at the Space Station Processing Facility (SSPF) of the KSC in Cape Canaveral, FL. Sterilization and planting of Arabidopsis thaliana seeds, Columbia-0 ecotype (Col-0), are described in detail in Dyachok et al. (2009). Briefly, sterilized seeds of wild-type were planted on growth media $(1.2 \%$ phyta-agar) consisting of $0.5 \mathrm{X}$ Murashige-Skoog (MS) salts and $1 \%$ sucrose (pH 5.7) layered onto $90 \times 90 \mathrm{~mm}$ square Petri dishes (Simport Scientific Inc., Beloeil, Quebec, Canada).

Immediately after planting, Petri dishes containing the seeds were exposed to FR light (3.2 $\mu \mathrm{mol} \mathrm{m} \mathrm{m}^{-2} \mathrm{~s}^{-1}$ ) for 10 minutes, using a specially constructed rectangular metal box (see Figure 1 and Results and Discussion for details). A single LED (ER-R2, 4606, Norlux Corporation, Carol Stream, IL) was used to provide FR illumination of the seeds inside the Petri dishes. Far-red treatment was carried out under dim light with the distance between the far-red LED and Petri dish set at $70 \mathrm{~mm}$. Petri dishes were immediately wrapped with a layer of aluminum foil after FR light treatment to keep seeds in complete darkness during imbibition. Petri dishes were then stored in a $4^{\circ} \mathrm{C}$ refrigerator. To activate the experiment and trigger germination, aluminum foil was removed and Petri dishes that contained seeds of Col-0 and 


\section{Lessons Learned from APEX 02-1 SVT and PVT}

act2 act8 double mutants (Nakashima et al., 2014) were installed on ABRS (Figure 2A). LEDs on ABRS were programmed to provide white light intensity at $120 \mu \mathrm{mol} \mathrm{m} \mathrm{m}^{-2}$ for a 14 hour $/ 10$ hour light/dark cycle, and temperature was maintained at $22 \mathrm{C}$.

In a parallel set of experiments, additional arabidopsis ecotypes, namely Landsberg (Ler), Wassilewskija (Ws) and C24, were tested for delayed germination after far-red light exposure. For this set of experiments, instead of using white light on ABRS, germination was triggered by transferring Petri dishes containing seed to a Conviron with $14 \mathrm{~h} / 10 \mathrm{~h}$ light/dark cycle and temperature set at $22 \mathrm{C}$. For each ecotype and treatment, percentage germination was calculated by visually inspecting plates under a stereomicroscope and counting the number of seeds where the radicle protruded from the seed coat. Percent germination was scored immediately after removal of aluminum foil and prior to transfer to the Conviron. Percent viability was scored by counting the number of germinated seed seven days after transfer to the Conviron. Thirty seeds were planted per plate.

\section{Light and Transmission Electron Microscopy (TEM)}

Seedlings grown in ABRS were harvested and fixed in KFTs preloaded with 3\% glutaraldehyde. After retrieval of seedlings from the KFTs, they were washed six times with phosphate-buffered saline (PBS), dehydrated in a graded ethanol series, and embedded in LR White resin (London Resin Co. Ltd., Reading, Berkshire, UK). The resin was then polymerized at $4^{\circ} \mathrm{C}$ under UV light for three days. Serial 0.25 and $0.1 \mu \mathrm{m}$ longitudinal sections were cut with a diamond knife and a Leica EM UC7 ultramicrotome (Leica Mikrosysteme $\mathrm{GmbH}$, Vienna, Austria) or RMC MT-X ultramicrotome (Boeckeler Instruments, Inc., Tucson, AZ). Semi-thin sections $(0.25 \mu \mathrm{m}$ in thickness) placed onto the slide glasses (Superfrost Plus; VWR International, LLC, Radnor, PA) were stained with $1 \%(\mathrm{w} / \mathrm{v})$ Toluidine Blue $\mathrm{O}$ (with $1 \%(\mathrm{w} / \mathrm{v})$ sodium borate) for five minutes and observed under a Nikon Microphot-2 (Nikon Corporation, Tokyo, Japan). Ultra-thin sections $(0.1 \mu \mathrm{m}$ in thickness $)$ were then put onto formvar-carbon-coated copper grids and stained with $2 \%$ uranyl acetate for 30 minutes, followed by incubation with Sato's lead for two minutes. Specimens were observed under a Zeiss 10A (Carl Zeiss AG, Oberkochen, Germany) or JEOL 2000-FX (JEOL, Tokyo, Japan) transmission electron microscope operated at $80 \mathrm{kV}$.

\section{Indirect Immunofluorescence Labeling}

Semi-thin sections $(0.25 \mu \mathrm{m}$ in thickness $)$ were cut with an ultramicrotome and mounted onto glass slides. Indirect immunofluorescence labeling was performed by applying and removing a series of $100 \mu \mathrm{L}$ droplets of the appropriate reagents to the sections as follows. Sections were blocked with $3 \%(\mathrm{w} / \mathrm{v})$ nonfat dry milk in PBS (Sigma-Aldrich, St. Louis, MO) for one hour and then washed with PBS for five minutes. Sections were incubated in hybridoma supernatant of the monoclonal antibody to a fucosylated xyloglucan (CCRC-M1; CarboSource Services, Athens, GA) diluted $1: 5$ in incubation buffer $[0.1 \% \mathrm{v} / \mathrm{v}$ goat serum, $0.8 \%$ BSA (Sigma-Aldrich, St. Louis, $\mathrm{MO}$ ), $0.1 \%$ ultrapure gelatin (USB Corporation, Cleveland, $\mathrm{OH}$ ), and two $\mathrm{mM}$ sodium azide (Sigma-Aldrich, St. Louis, MO)] for 16 to 18 hours at $4^{\circ} \mathrm{C}$. Sections were then washed with PBS three times for five minutes each, and goat anti-mouse IgG conjugated with Alexa-Fluor 488 (Molecular Probes, Life Technologies Corporation, Carlsbad, CA) diluted 1:100 in incubation buffer was applied for 90 to 120 minutes at room temperature in the dark. Sections were then washed with PBS three times for five minutes and distilled water for an additional five minutes. Sections were mounted on coverslips using Citifluor antifade AF1 (Electron Microscopy Sciences, Hatfield, PA). A Leica TCS SP2 AOBS Confocal Laser Scanning Microscope (Leica Microsystems CMS GmbH, Mannheim, Germany) was used to image Alexa Fluor 488.

\section{RESULTS AND DISCUSSION}

\section{Delaying Arabidopsis Seed Germination for Spaceflight Studies}

Prior to SVT and PVT, we defined several success criteria to determine flight readiness of APEX 02-1. SVT and PVT focused on two success criteria that other spaceflight investigators might find useful. First, we evaluated a system to 


\section{Lessons Learned from APEX 02-1 SVT and PVT}

delay arabidopsis seed germination until installation on ABRS. Second, we assessed the quality of seedling fixation on KFTs for postflight microscopic analyses. The former criterion was important to ensure that seedling development occurred only in microgravity and therefore minimized any additional source of variation that could complicate the interpretation of science results. Furthermore, delaying seed germination until the experiment was activated on ABRS eliminated the need for replanting in case the launch was postponed by several days or weeks. The ability to delay seed germination could also provide the ISS crew flexibility in regard to timing Petri plate installation on ABRS.

A simple protocol to delay arabidopsis seed germination for APEX 02-1 was designed based on classic experiments with lettuce seed. In lettuce, germination is triggered by red (R) light while FR light reverses R-light-induced germination (Borthwick et al., 1954). This R-FR light, reversibility of lettuce seed germination is known to involve the photoreceptor, phytochrome (for review, see Wang and Deng, 2004). Because the R-FR reversibility of germination can also occur in arabidopsis (Oh et al., 2004), we tested whether imbibing arabidopsis seed exposed briefly to FR light can be kept in a dormant state until they are exposed to light on the ABRS hardware. A portable light-tight metal box was constructed to implement FR light treatment of seed at KSC Space Station Processing Facility (SSPF). This box consisted of a single far-red LED (3.2 $\mu \mathrm{mol} \mathrm{m} \mathrm{m}^{-2} \mathrm{~s}^{-1}$ intensity) which could accommodate one $9 \mathrm{~cm}$ by $9 \mathrm{~cm}$ square Petri dish (see Figure 1 for details). During PVT, arabidopsis seeds planted on phyta-agar growth media in Petri dishes were immediately exposed to FR light for 10 minutes. Petri dishes were then wrapped with a single layer of aluminum foil and kept in a $4^{\circ} \mathrm{C}$ refrigerator for three days. On day three, aluminum foil was removed and Petri dishes were installed on ABRS. The camera system on the green fluorescent protein (GFP) imaging system (GIS) (Paul et al., 2013) of ABRS was used to document seedling growth (Inset in Figure 2A). It was found that FR-treated seed did not germinate when kept in the cold and in the dark (Figure 2B). Within two days of installation on ABRS, primary roots were visible (Figure 2C) and after seven days, seedling growth was robust
(Figure 2D). Our observations indicated that the custom-made FR light box described in Figure 1 was an effective and convenient method for controlling the timing of arabidopsis seed germination for plant space biology studies using ABRS.

Because the time allotted for evaluating seed germination using the FR light box on the ABRS unit during SVT and PVT was limited, we conducted more thorough laboratory testing to verify the effectiveness of the FR light treatment to delay arabidopsis seed germination. In addition to Col-0, we evaluated three other ecotypes, namely C24, Ler and, Ws. One set of Petri dishes planted with the different ecotypes was exposed to FR light for 10 minutes using the light box described in Figure 1, while another set was not treated. After FR light treatment, Petri dishes were wrapped with aluminum foil and divided further into two sets. To test whether maintaining FRtreated seed in the cold is required to keep seed in a dormant state, one set of Petri dishes was stored in a $4^{\circ} \mathrm{C}$ refrigerator and one set was kept at room temperature. After two or 10 weeks, aluminum foil was removed and the percentage of germinating seed was recorded. To ensure that FR treatment did not compromise seed viability, percent germination was again scored seven days after exposure to white light in the Conviron. These numbers are reflected as percent viability in Table 1.

Consistent with our PVT results, FR light treatment prevented seed germination in ecotype Col-0 for up to 10 weeks of storage at $4^{\circ} \mathrm{C}$. In Col-0 samples not FR-treated, more than $75 \%$ of seed germinated within two weeks, regardless of temperature storage conditions (Table 1). Interestingly, the delay in germination in FRtreated Col-0 seeds was also observed when Petri dishes were stored in the dark at room temperature. This indicated that germination of Col-0 seed treated with FR light can be delayed without the need for cold storage (Table 1). FR treatment was also effective in delaying seed germination in Ler ecotype, regardless of whether Petri dishes were stored at $4^{\circ} \mathrm{C}$ or room temperature (Table 1). In the Ler ecotype stored in the dark at $4^{\circ} \mathrm{C}$, however, a higher percentage of seed germinated after 10 weeks of storage compared to Col-0, indicating a slight variability in the FR response among different ecotypes. 
Although germination of ecotype C24 could also be delayed by FR light treatment, it appeared that such a treatment was more effective on $\mathrm{C} 24$ seeds if they were kept in the dark and maintained at room temperature. If FR-light-treated $\mathrm{C} 24$ seeds were kept at $4^{\circ} \mathrm{C}$, more than $85 \%$ of the seeds germinated within two weeks, whereas only $7 \%$ germinated if they were kept at room temperature (Table 1). Our data also showed that simply keeping seed in the dark is sufficient to delay germination for the Ws ecotype (Table 1).
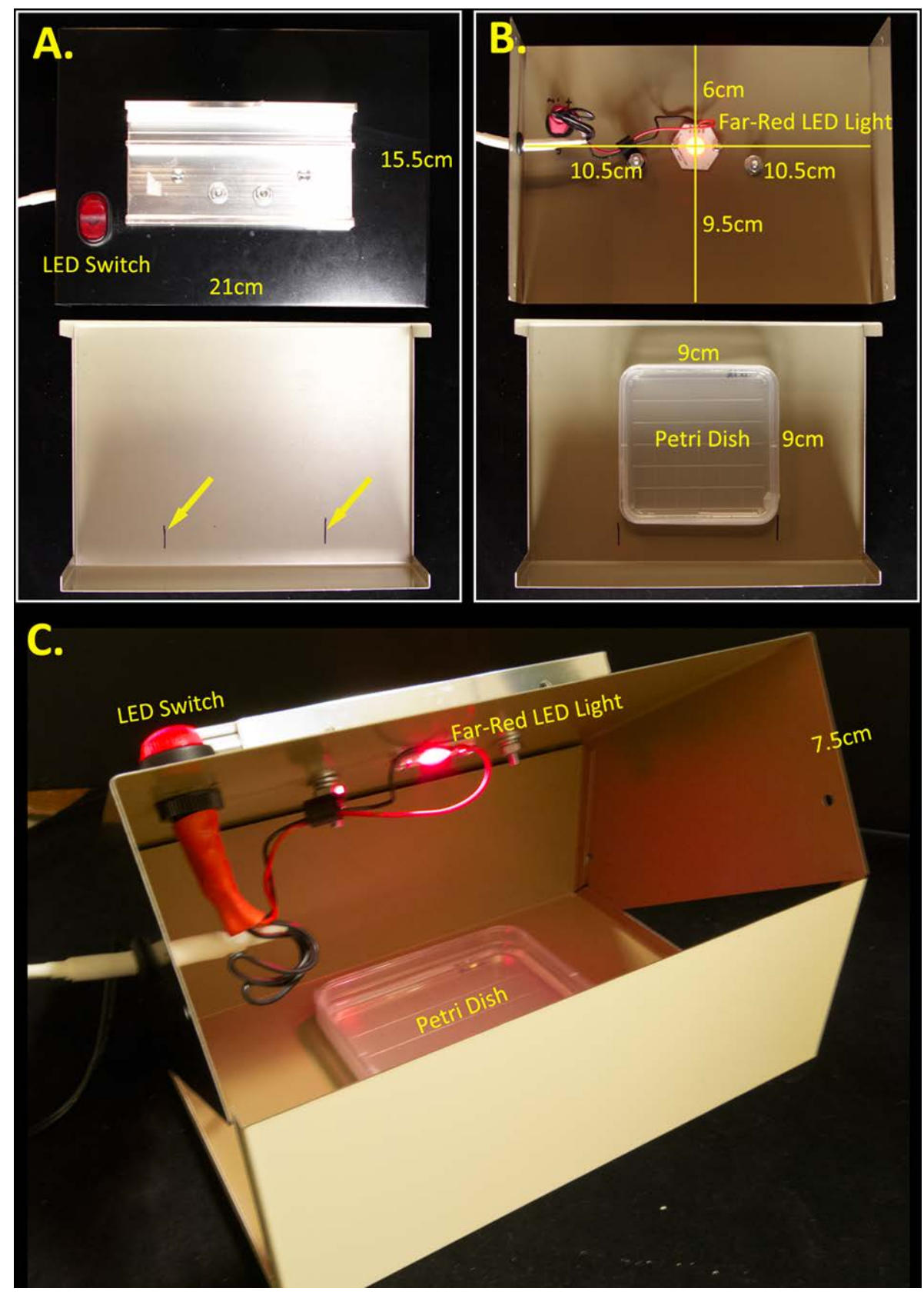

Figure 1. FR metal light box for delaying arabidopsis seed germination. (A) The light box consists of two parts. The lid, which contains the LED switch at the roof of the box, and a base where the Petri dish is positioned (arrows). (B) The ceiling of the metal lid showing the location of the FR LED, and the base showing placement of a Petri dish for FR light exposure. (C) Assembly of the metal box for exposing Petri dishes containing arabidopsis seeds to FR light. A 10-minute FR light treatment using this device, followed by maintaining plates in complete darkness, delayed seed germination until seeds were exposed to white light on ABRS. 


\begin{tabular}{|c|c|c|c|c|c|c|c|c|}
\hline \multirow[b]{4}{*}{ Ecotype } & \multicolumn{8}{|c|}{2 Weeks } \\
\hline & \multicolumn{4}{|c|}{ Non-treated } & \multicolumn{4}{|c|}{ Far-Red Light Treatment } \\
\hline & \multicolumn{2}{|c|}{ Room Temperature } & \multicolumn{2}{|c|}{$4^{\circ} \mathrm{C}$} & \multicolumn{2}{|c|}{ Room Temperature } & \multicolumn{2}{|c|}{$4^{\circ} \mathrm{C}$} \\
\hline & Germination $(\%)^{*}$ & Viability $(\%)^{* *}$ & Germination $(\%)^{*}$ & Viability $(\%)^{* *}$ & Germination $(\%)^{*}$ & Viability $(\%)^{* *}$ & Germination $(\%)^{*}$ & Viability $(\%)^{* *}$ \\
\hline Col-0 & 100 & 100 & 76.7 & 100 & 0 & 100 & 3.3 & 100 \\
\hline $\mathrm{C} 24$ & 23.3 & 86.7 & 90 & 93.3 & 6.7 & 96.7 & 86.7 & 100 \\
\hline Ler & 63.3 & 96.7 & 23.3 & 96.7 & 0 & 96.7 & 16.7 & 100 \\
\hline Ws & 0 & 86.7 & 10 & 100 & 3.3 & 86.7 & 6.7 & 100 \\
\hline
\end{tabular}

10 Weeks

\begin{tabular}{|c|c|c|c|c|c|c|c|c|}
\hline \multirow[b]{3}{*}{ Ecotype } & \multicolumn{4}{|c|}{ Non-treated } & \multicolumn{4}{|c|}{ Far-Red Light Treatment } \\
\hline & \multicolumn{2}{|c|}{ Room Temperature } & \multicolumn{2}{|c|}{$4^{\circ} \mathrm{C}$} & \multicolumn{2}{|c|}{ Room Temperature } & \multicolumn{2}{|l|}{$4^{\circ} \mathrm{C}$} \\
\hline & Germination (\%)* & Viability $(\%)^{* *}$ & Germination (\%)* & Viability $(\%)^{* *}$ & Germination $(\%)^{*}$ & Viability $(\%)^{* *}$ & Germination $(\%)^{*}$ & Viability $(\%)^{* *}$ \\
\hline Col-0 & 93.3 & 100 & 100 & 100 & 3.3 & 76.7 & 3.3 & 100 \\
\hline $\mathrm{C} 24$ & 26.7 & 80 & 100 & 100 & 3.3 & 50 & 93.3 & 100 \\
\hline Ler & 63.3 & 93.3 & 70 & 90 & 13.3 & 96.7 & 50 & 96.7 \\
\hline Ws & 0 & 100 & 20 & 96.7 & 3.3 & 96.7 & 3.3 & 100 \\
\hline
\end{tabular}

*Indicates the percent of seeds that germinated before exposure to white light.

$* *$ indicates the final percentage of seed that germinated after exposure to white light.

Table 1. Impact of FR light treatment on germination and viability of four Arabidopsis ecotypes (Col-0, C24, Ler, and Ws). Percent germination and viability were obtained from a total of 30 seeds planted per ecotype and treatment regime.

Interestingly, we found that the prolonged absence of light in C24 seed treated with FR light and stored at room temperature compromised seed viability. Cold storage of C24 seed after FR light exposure appears to maintain viability (Table 1). Taken together, our results indicated that FR light treatment followed by storage in darkness was most effective for delaying seed germination in ecotype Col-0 and, to a lesser extent, Ler. For Ws, delay in germination could be accomplished by simply withholding light. In ecotype $\mathrm{C} 24$, the control of seed germination appeared to be more complex and involved the interplay between both, low temperature and light conditions. Although germination and viability tests for all four genotypes reported in Table 1 was conducted only once due to the large number of plates that had to be treated, the percentages obtained for the Col-0 ecotype, which is the main ecotype for APEX 201 , was repeated at least four times and showed consistent results.

\section{Root Morphology and Cell Wall Antigenicity of ABRS-Grown Arabidopsis Seedlings during SVT}

For APEX 02-1, we proposed to conduct light microscopy and transmission electron microscopy
(TEM) to determine how microgravity impacts cellular structure and correlate any changes in cellular structure with modified patterns of gene expression. Some of the proposed microscopy experiments would involve immunolabeling of cell wall antigens in root sections using a suite of monoclonal antibodies (Pattathil et al., 2010). Thus, a second criterion we paid careful attention to during SVT was the quality of seedling fixation on KFTs. In light micrographs of seedlings fixed in KFTs, general root cellular structure appeared to be poorly preserved. Toluidine-blue-O-stained root sections fixed in KFTs during SVT, for example, were smeared as opposed to the crisp bright blue and purple color from root sections fixed using a procedure that we optimized after PVT (compare Figure 3A, B to Figure 7G). Details of the fixation protocol to which SVT and PVT results were compared are described in detail below (Figures 6 and 7). Cytosolic components of roots from SVT also appeared degraded, and plasmolysis was rampant in the root transition zone of the primary root (Figure 3A, B). The poor fixation of seedlings during SVT was also apparent from indirect immunofluorescence labeling of root sections with fucosylated xyloglucan antibodies (CCRC-M1; Pattathil et al., 2010). Although cell wall antigenicity was 
preserved, fluorescence was mostly grainy (Figure 3C, D). At the TEM level, samples from SVT exhibited irregularly shaped nuclei and a significant amount of vacuolation in cells of the root transition zone (Figure 3E, F).
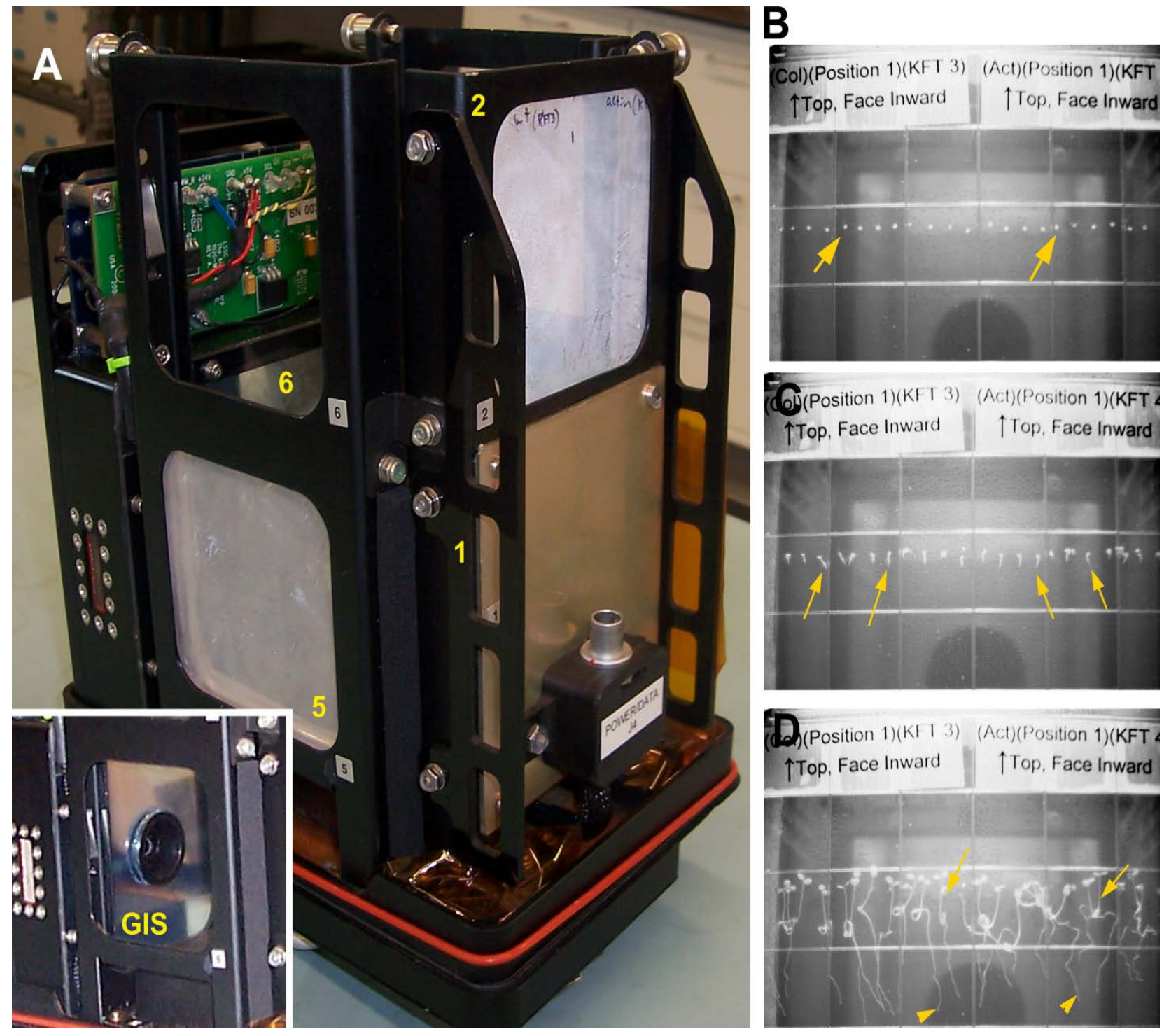

Figure 2. ABRS and images of Petri dish on ABRS-GIS. (A) ABRS showing location of Petri dishes. Four out of six positions are indicated as yellow numbers $(1,2,5,6)$. (B) Petri dish in position one of ABRS three days after planting (day 0 of installation on ABRS). Images of dishes were captured using the GIS unit (inset in A) of ABRS. Note that seeds have not yet germinated (arrows). (C) At two days after installation on ABRS, radicles can be seen protruding (arrows). (D) At seven days after installation on ABRS, seedlings have extended hypocotyls (arrows) and primary roots (arrowheads). Two genotypes (wild-type Col-0 and act2 act8 double mutant) were planted on the same plate during SVT and PVT. Note that at seven days after installation on ABRS, the act2 act8 double mutants, located on the right half of the plate (D), have primary roots with more wavy growth patterns. 

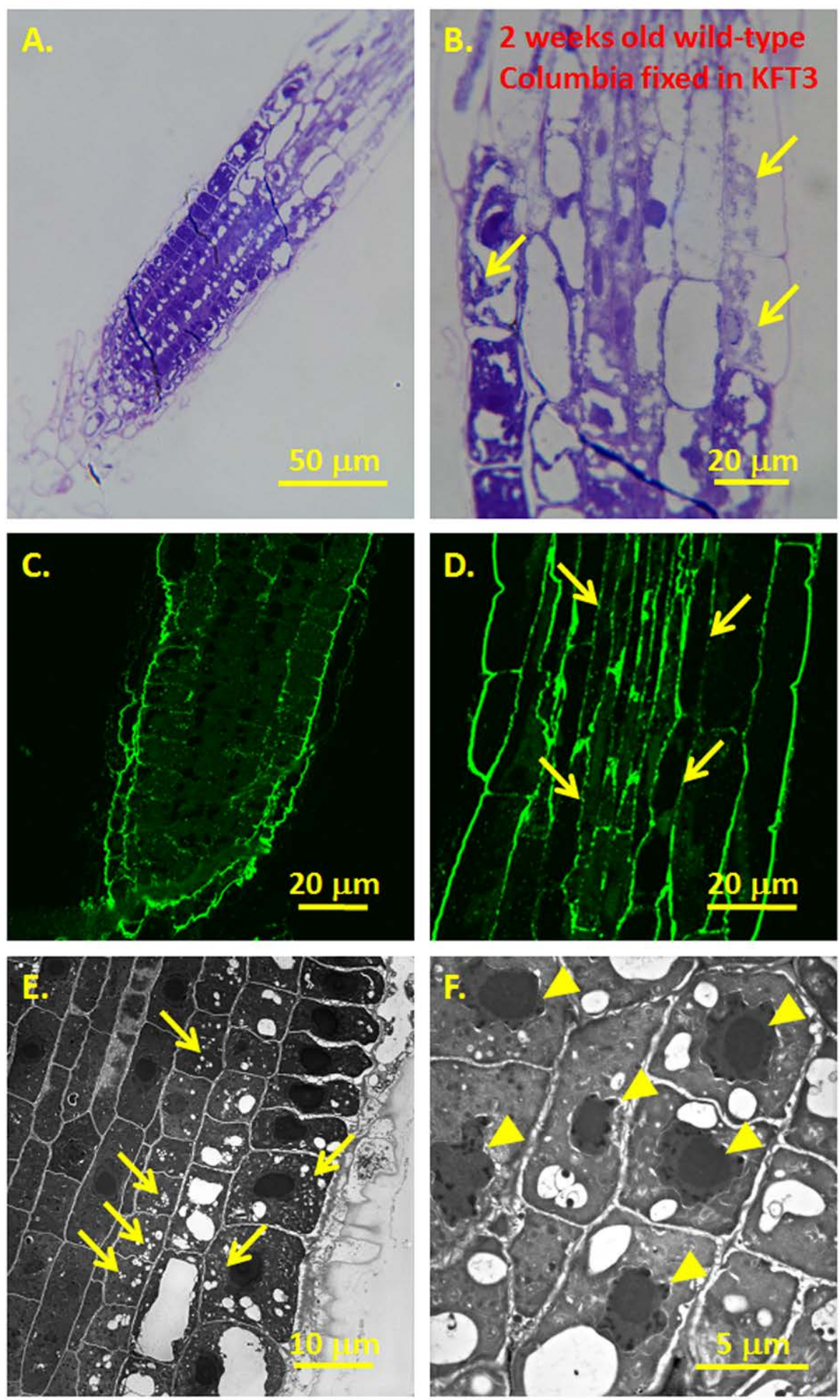

Figure 3. Quality of fixation of arabidopsis roots during SVT. (A, B) Semi-thin sections stained with Toluidine Blue $O$. Note that some root cells in the transition zone were plasmolyzed (arrows). (C, D) Roots labeled with monoclonal antibodies against fucosylated xyloglucan (CCRC-M1). Note the grainy fluorescence patterns and occurrence of punctate structures (arrows) in panels $C$ and $D$. (E, F) TEM micrographs from the root transition zone. Note the extensive number of vacuoles (arrows) and irregularly shaped nuclei (arrowheads). Each set of micrographs are representative of four individual roots. 
A number of observations could explain the poor quality of cell preservation during SVT. First, the potency of the glutaraldehyde fixative might have been compromised due to oxidization while stored on KFTs. As shown in Figure 4, fixative was in contact with a small volume of air after KFT actuation that might have contributed to more rapid oxidation of the fixative. Second, fixative penetration into the tissues might not have been quick enough. Protocols for optimal chemical fixation used in the laboratory are typically accompanied by vacuum infiltration to allow more rapid fixative penetration (Russin and Trivett, 2001). Because vacuum infiltration was not possible in KFTs, fixative penetration could have been inefficient. Third, seedlings were stored in fixatives for an extended period of time (overfixation) compared to what is normally done during routine laboratory procedures. The extended time of seedlings on fixative could have affected overall root cellular structure.

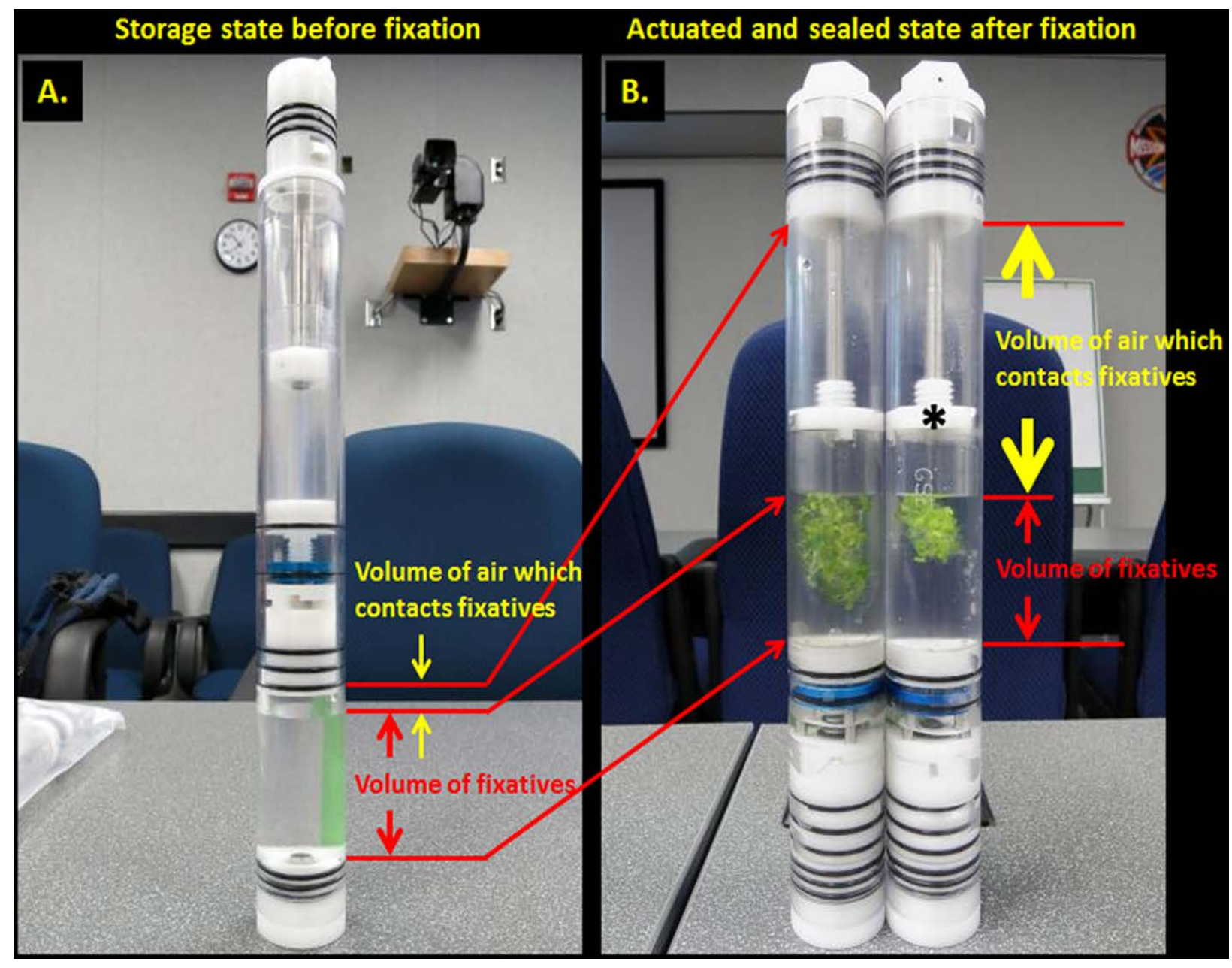

Figure 4. KFTs preloaded with fixative used for in-orbit chemical fixation and containment of biological samples. (A) KFTs before actuation. The volume of fixative (red lines) is shown relative to the volume of air (yellow). (B) After actuation, the volume of air in contact with fixative increased. This could contribute to oxidation of the fixative. If an extra O-ring is added on the KFTs at the position indicated by the asterisk in panel $B$, the volume of air which potentially contacts fixative after the actuation would be reduced. This may lead to improvement in the quality of fixation for future microscopy work involving KFTs. 


\section{Resolving Fixation Problems Encountered during SVT}

Poor fixation on KFTs during SVT prompted us to make a few minor adjustments to our fixation schemes during PVT. As noted, a possible contributor to poor fixation was exposure of glutaraldehyde to air in the KFT for prolonged periods, particularly after actuation (Figure 4). To minimize any adverse effect that this might have on fixation quality, the following procedures to handle fixative solution were followed for PVT. First, we increased the glutaraldehyde concentration from $3 \%$ to $5 \%$ to account for potential loss in potency. Second, before fixative was loaded into KFTs, $\mathrm{N}_{2}$ gas was bubbled over the glutaraldehyde solution in an effort to reduce fixative oxidation. Third, we stored KFTs at $4^{\circ} \mathrm{C}$ and in the dark immediately after loading fixative and after seedling fixation. These changes are summarized in Figure 5.
After implementing the changes in Figure 5, seedlings were processed in a similar manner to those from SVT and root cell structure was evaluated by light microscopy and TEM. We found that implementation of new fixative handling protocols improved the quality of fixation during PVT. For instance, ToluidineBlue-O-stained semi-thin root sections displayed thin bright blue and purple coloration, as opposed to the smeared patterns observed during SVT (compare Figure 6A, B to Figure 3A, B). Moreover, fluorescence of antibody-tagged fucosylated xyloglucans from semi-thin root sections was crisp and smooth in PVT, compared to grainy patterns observed during SVT (compare Figure 6C, D to Figure 3C, D). However, we still observed plasmolysis in cells of the root transition zone, and cells appeared to be highly vacuolated (Figure 6A, C). The problem of plasmolysis might be difficult to overcome given the need to store samples in fixative for an extended period of time.
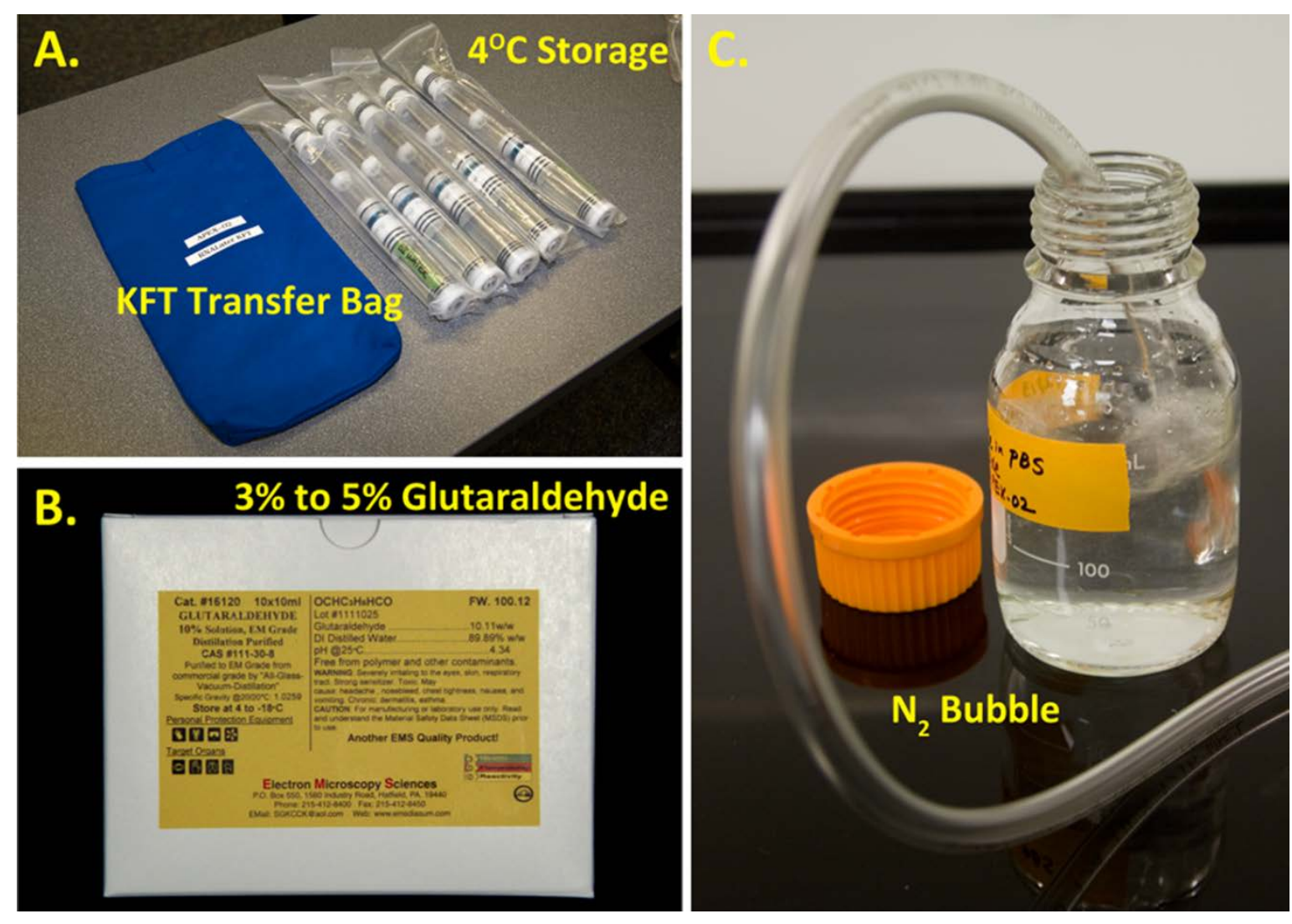

Figure 5. Adjustments made in composition and handling of fixative during PVT. Adjustments made included storing KFTs at $4^{\circ} \mathrm{C}$ and in the dark, both prior to fixation and after actuation (A); increasing glutaraldehyde concentration from $3 \%$ to $5 \%(B)$; and bubbling $\mathrm{N}_{2}$ gas over freshly prepared glutaraldehyde solution $(\mathbf{C})$. 

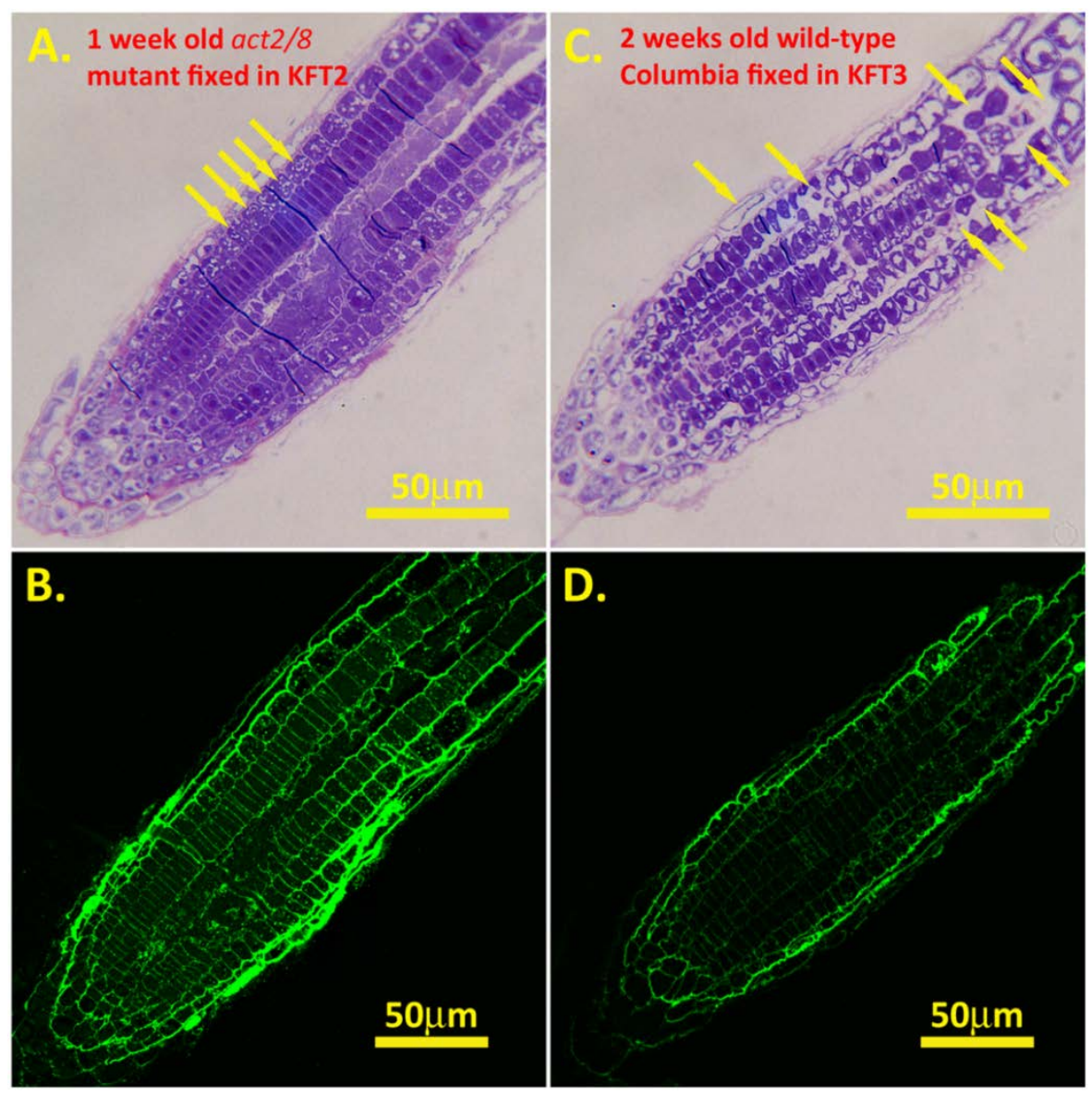

Figure 6. Quality of seedling fixation during PVT. Serial semi-thin sections $(0.25 \mu \mathrm{m}$ in thickness) taken from a median longitudinal section of an arabidopsis primary root from seedlings grown in ABRS hardware and fixed in KFTs were stained with Toluidine Blue $O$ ( $A$ and $C$ ) and processed by indirect immunofluorescence using a monoclonal antibody against fucosylated xyloglucan (CCRC-M1) (B and D). Note vacuolation (arrows in A) and plasmolysis (arrows in C) in Toluidine-Blue-O-stained roots. Each set of micrographs are representative of three individual roots.

\section{Additional Steps to Optimize Seedling Fixation in Aldehydes for Microscopy Studies}

Although the minor adjustments implemented during PVT led to a general improvement in fixation quality, some notable problems persisted, including the induction of highly vacuolated cells in the root transition zone (Figure 6). Vacuolated cells could be indicative that extensive dehydration might have been occurring while seedlings were exposed for an extended period to fixative while in KFTs. The fact that glutaraldehyde in the fixative was increased to $5 \%$ might have aggravated this problem. We therefore performed additional tests that roughly mirrored the duration of fixation on KFTs during SVT and PVT but, at the same time, considered modifying the composition of the fixative solution. For these additional tests, we used Karnovsky's fixative (Karnovsky, 1965), which consisted of 4\% paraformaldehyde and 3\% glutaraldehyde.

Karnovsky's fixative was used in combination with the new protocols for fixative treatment that we implemented during PVT (e.g., bubbling of $\mathrm{N}_{2}$ gas, and cold and dark storage of fixative; see Figure 5). We found that the use of Karnovsky's fixative led to further improvements in the quality of fixation. For example, the thin bright blue and purple color of Toluidine-Blue-O-stained roots was predominant, and the fluorescence of antibody-tagged fucosylated xyloglucans from semi-thin root sections was crisp and smooth (Figure 7B, E). More importantly, the vacuolation and plasmolysis that was observed during SVT 
and PVT was minimal (Figure 7A, D). Taken together, these results indicated that Karnovsky's fixative in, combination with the improved fixative handling procedures implemented in Figure 5, might be the best system to use for the actual APEX 02-1 experiments. The minimal plasmolysis and vacuolation observed during these additional tests could be due in part to the inclusion of paraformaldehyde in the fixative. Although its ability to cross-link proteins is less than glutaraldehyde, paraformaldehyde penetrates tissues more quickly because of its smaller molecular size and thus could minimize the problem of slow fixative penetration (Paljarvi et al., 1979).
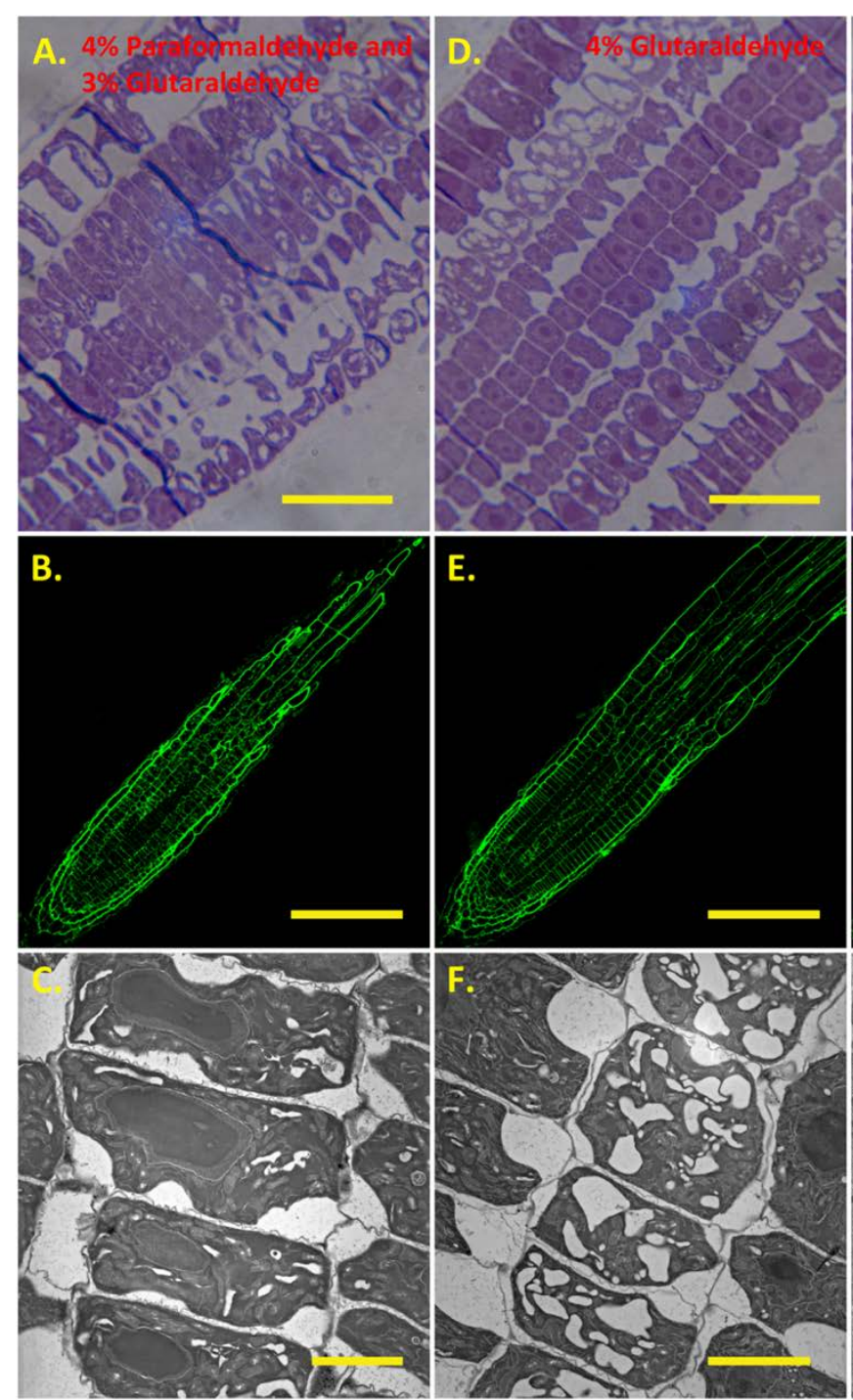
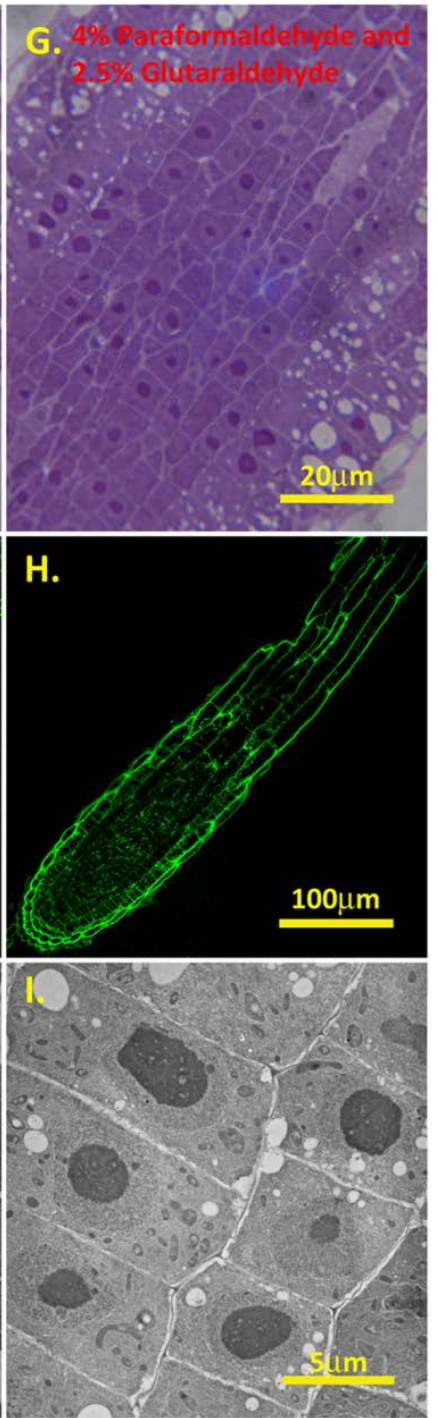

Figure 7. Additional tests to optimize seedling fixation in aldehyde for microscopy studies. Serial semi-thin sections $(0.25 \mu \mathrm{m}$ in thickness) taken from a median longitudinal section of an arabidopsis primary root from seedlings fixed at the laboratory that mirrored the duration of fixation on KFTs during SVT and PVT. Fixation was conducted with $4 \%$ paraformaldehyde and $3 \%$ glutaraldehyde (A to C), and $4 \%$ glutaraldehyde (D to F). Sections were stained with Toluidine Blue $O$ (A and D) and indirect immunofluorescence labeling with monoclonal antibody against fucosylated xyloglucan (CCRC-M1) (B and E), and ultra-thin sections were observed under the transmission electron microscope ( $C$ and F). For comparison, a similar set of seedlings was fixed in $4 \%$ paraformaldehyde and $2.5 \%$ glutaraldehyde, and processed using routine laboratory protocols (e.g., no extended fixation) (G to I). Despite the slight plasmolysis and vacuolation, fixation quality with paraformaldehyde in the fixative generally improved. Each set of micrographs are representative of three individual roots. 


\section{Lessons Learned from APEX 02-1 SVT and PVT}

Based on our SVT and PVT, there are additional avenues by which chemical fixation of plant samples in space for structural studies can be improved. For such improvements to be made, one must look at the design of the KFT. For instance, the volume of air that contacts the fixative increased after KFT actuation (see Figure 4). As noted, this increased air volume could hasten fixative oxidation. It might be possible to minimize this problem with a minor design change in the KFT where an extra O-ring can be added to reduce the volume of air that comes in contact with fixative after actuation (Figure 4).

Another way to improve chemical fixation would be to include a low concentration of detergent, such as $0.1 \%(\mathrm{v} / \mathrm{v})$ Triton-X 100 or Tween 20 in the fixative. The detergents would make the sample surface more hydrophilic, allowing it to completely immerse into the fixative solution. Addition of detergent would ensure that fixation is rapid and complete. However, because detergents have not been certified to fly in KFTs, the need to completely submerge seedlings in the fixative and shake KFTs occasionally within the first few hours of actuation to facilitate fixation will be important to emphasize during crew training.

\section{CONCLUSIONS}

During APEX 02-1 SVT and PVT, we designed a portable metal box for FR illumination that would enable us to delay arabidopsis seed germination until installation on ABRS. The short FR light treatment did not compromise seed viability and could potentially delay germination for up to 10 weeks in the arabidopsis Col-0 ecotype. Through SVT and PVT, we have also determined an optimal fixative composition, as well as additional ways to handle fixative prior to loading on KFTs. Flexibility in conducting space biology experiments is often dictated by hardware design and established operating procedures specified by NASA because of safety and other logistical issues. Lessons learned from SVT and PVT, which typically are not shared with the scientific community, can be valuable to increase the likelihood that the quality of plant material obtained from spaceflight is not compromised.

\section{ACKNOWLEDGEMENTS}

This work was supported by NASA grant NNX12AM94G. We thank Dr. Howard Levine, Bryan Onate, Jose Camacho, Stacy Engel, Susan Manningroach, David Reed, and many dedicated professionals at the Kennedy Space Center for their excellent technical support. We also thank Terry McKeown, Noble Foundation, for constructing the FR light box used for our germination tests; Dr. Karl Hasenstein, University of Louisiana, for advice on how to handle aldehyde-based fixative for spaceflight experiments; and Dr. Enamul Huq, University of Texas, for the suggestion to use FR light to control germination. We are also grateful to Dr. Robert Ferl and Dr. Anna-Lisa Paul, University of Florida, for sharing their experiences on the intricacies of conducting space plant biology research. Finally, we thank Jackie Kelley (Noble Foundation) for additional editing of the manuscript.

\section{REFERENCES}

Borthwick HA, Hendricks SB, Toole EH, Toole VK (1954) Action of light on lettuce-seed germination. Botanical Gazette 115: 205-225

Dyachok J, Yoo CM, Palanichelvam K, Blancaflor EB (2009) Sample preparation for fluorescence imaging of the cytoskeleton in fixed and living plant roots. In Cytoskeleton Methods and Protocols: Methods in Molecular Biology Series, R.H. Gavin (ed), pp 157-169. Totowa, NJ: Humana Press Inc.

Karnovsky MJ (1965) A formaldehydeglutaraldehyde fixative of high osmolality for use in electron microscopy. Journal of Cell Biology 27: 137A-138A

Levine HG, Cox DR, Reed DW, Mortenson TE, Shellack JL, Wells HW, Murdock T, Regan MF, Albino SA, Cohen J (2009) The advanced biological research system (ABRS): A single middeck payload for conducting experimentation on the International Space Station. In Proceedings of the 47th AIAA Aerospace Sciences Meeting Including The New Horizons Forum and Aerospace Exposition, 5-8 January 2009, Orlando, FL, pp $1-10$ 


\section{Lessons Learned from APEX 02-1 SVT and PVT}

Millar KD, Johnson CM, Edelmann RE, Kiss JZ (2011) An endogenous growth pattern of roots is revealed in seedlings grown in microgravity. Astrobiology 11: 787-797

Nakashima J, Liao F, Sparks JA, Tang Y, Blancaflor EB (2014) The actin cytoskeleton is a suppressor of the endogenous skewing behavior of Arabidopsis primary roots in microgravity. Plant Biology 16: 142-150

Oh E, Kim J, Park E, Kim JI, Kang C, Choi G (2004) PIL5, a phytochrome-interacting basic helix-loop-helix protein, is a key negative regulator of seed germination in Arabidopsis thaliana. Plant Cell 16: 3045-3058

Paljarvi L, Garcia JH, Kalimo H (1979) The efficiency of aldehyde fixation for electron microscopy: stabilization of rat brain tissue to withstand osmotic stress. Histochemical Journal 11: 267-276

Pattathil S, Avci U, Baldwin D, Swennes AG, McGill JA, Popper Z, Bootten T, Albert A, Davis RH, Chennareddy C, Dong R, O'Shea B, Rossi R, Leoff C, Freshour G, Narra R, O'Neil M, York WS, Hahn MG (2010) A comprehensive toolkit of plant cell wall glycan-directed monoclonal antibodies. Plant Physiology 153: 514-525
Paul A-L, Amalfitano AE, Ferl RJ (2012) Plant growth strategies are remodeled by spaceflight. BMC Plant Biology 12: 232-246

Paul A-L, Levine HG, McLamb W, Norwood KL, Reed D, Stutte GW, Wells HW, Ferl RJ (2005) Plant molecular biology in the space station era: utilization of KSC fixation tubes with RNAlater. Acta Astronautica 56: 623628

Paul A-L, Zupanska AK, Schultz ER, Ferl RJ (2013) Organ-specific remodeling of the Arabidopsis transcriptome in response to spaceflight. BMC Plant Biology 13: 112-122

Russin WA, Trivett CL (2001) Vacuummicrowave combination for processing plant tissues for electron microscopy. In Microwave Techniques and Protocols, R.T. Giberson, and R.S. Demaree Jr. (ed), pp 25-35. Totowa, NJ: Humana Press Inc.

Ruyters G, Braun M (2014) Plant biology in space: recent accomplishments and recommendations for future research. Plant Biology 16: 4-11

Wang H, Deng XW (2004) Phytochrome signaling mechanism. The Arabidopsis Book. 3: $\mathrm{e} 0074$. 